\title{
Case Report \\ The Cervical Anterior Approach for the Resection of Superior Posterior Neurogenic Tumor: A Case Report
}

\author{
Motoki Sakuraba, MD,PhD, ${ }^{1}$ Yoshikazu Miyasaka, $\mathrm{MD},{ }^{2}$ \\ Yoshiki Kodu, MD, ${ }^{2}$ and Kenji Suzuki, MD $^{2}$
}

\begin{abstract}
Many approaches for resection of the superior mediastinal tumors have been reported. We introduce an approach, which we call the cervical anterior approach. This approach is only cervical and does not require a sternotomy. Merits of this approach include the ability to remove the tumor without opening the mediastinal or parietal pleura, as well as obviating draining the thoracic cavity. The tumor is also directly visible, and the surgeon can avoid injury to the great vessels. This approach is recommended when the tumor is located superior to the third thoracic vertebra level, when it borders the great vessels, and when it does not border the trunk of the brachial plexus or nerve root. This approach is easy and safe for surgical procedures.
\end{abstract}

Keywords: neurogenic tumor, superior posterior mediastinum

\section{Introduction}

Many approaches for the resection of superior posterior mediastinal tumors have been reported. Each technique has distinct advantages and disadvantages with the location of the tumor often dictating the operative approach. We introduce the cervical anterior approach for the resection of superior posterior neurogenic tumors.

\section{Case}

A 41-year-old male presented with an abnormal shadow in the left apex of his lung two years prior. He

${ }^{1}$ Division of General Thoracic Surgery, Juntendo University Nerima Hospital, Tokyo, Japan

${ }^{2}$ Department of General Thoracic Surgery, Juntendo University School of Medicine, Tokyo, Japan

Received: January 18, 2011; Accepted: February 21, 2011 Corresponding author: Motoki Sakuraba, MD. Division of General Thoracic Surgery, Southern Tohoku Group Tokyo Hospital, 3-5-2 Egota, Nakano-ku, Tokyo 165-0022, Japan

Email: m-sakuraba@hotmail.co.jp

(C)2011 The Editorial Committee of Annals of Thoracic and Cardiovascular Surgery. All rights reserved. was admitted to our hospital because the mass had grown based on chest X-ray (Fig. 1A); however, he had no neurogenic symptoms. Chest computed tomography scan and magnetic resonance imaging revealed a left superior mediastinal mass at the Th1 to Th3 level, behind the left subclavian artery and left vertebral artery, measuring $40 \mathrm{~mm}$ in size, which was suspected to be a neurogenic tumor (Fig. 1B). Based on the relation of the vessels to the tumor, we selected a cervical anterior approach (Fig. 2A). General anesthesia was administered with the patient in the supine position with the head turned to the right. A left-sided collar incision was performed, and the platysma was cut. The left sternocleidomastoid muscle was isolated and retracted laterally, while the anterior cervical muscles were retracted medially. The middle thyroid was isolated and ligated. Next, in order to avoid injury to the left recurrent laryngeal nerve, the left common carotid artery was retracted laterally while the trachea with its associated fatty tissue which included the left recurrent laryngeal nerve was retracted medially. In this matter, we reached the left edge of the esophagus. When the esophagus with the trachea and fatty tissue was retracted medially, we could see the longus colli muscle inserting onto the vertebra as well as tumor (Fig. 2B). It was then possible to 

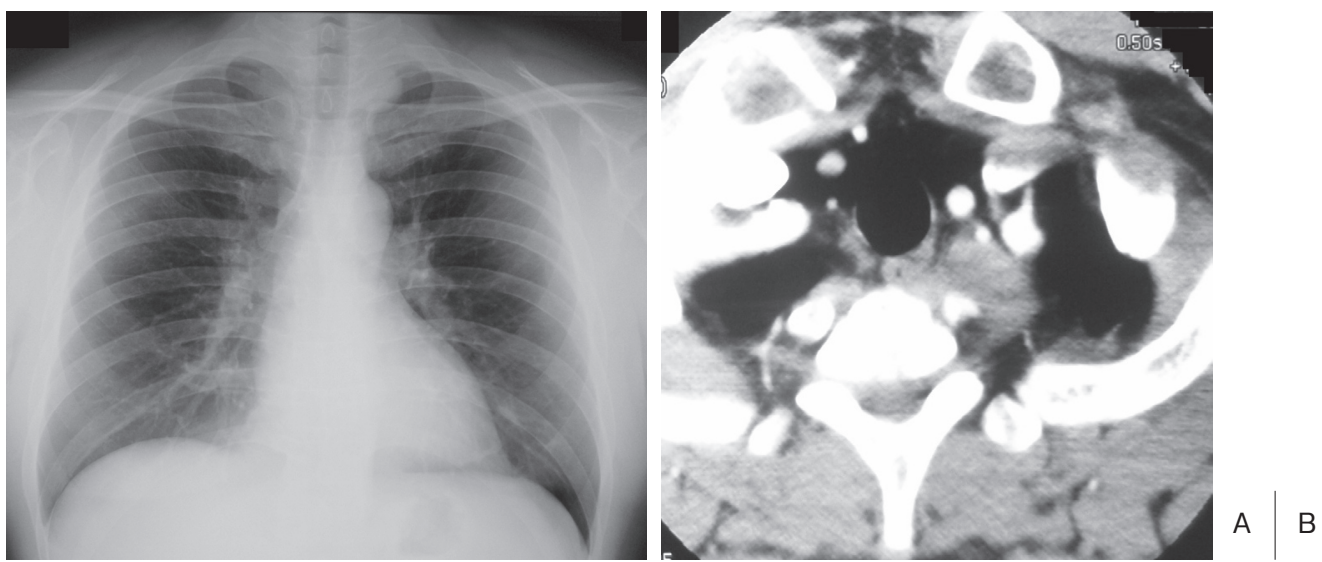

Fig. 1

A) Chest X-ray shows the tumor located in the left apical lung field.

B) Chest computed tomography scan shows a left superior mediastinal mass measuring $40 \mathrm{~mm}$ in size, behind the left subclavian and vertebral arteries.

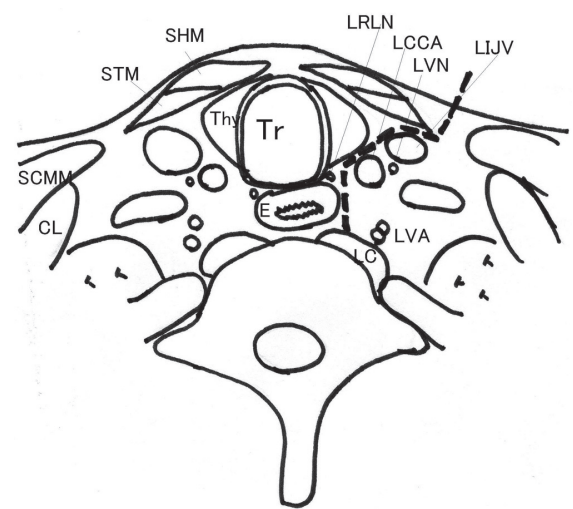

Fig. 2

A) Schema of the cervical anterior approach. The dotted line shows the divided line.

Tr: trachea; Thy: thyroid; SHM: sternohyoid muscle; STM: sternothyroid muscle; SCMM: sternocleidmastoid muscle; CL: clavicle; E: esophagus; LRLN: left recurrent lalyngeal nerve; LCCA: left common carotid artery; LIJV: left internal jugular vein; LVN: left vagus nerve; LVA: left vertebral artery; LC: longus colli muscle

B) Operative findings demonstrate that the tumor is divided by the isolated line. E: esophagus; T: tumor; L: longus colli muscle; Tr: trachea

C) Operative findings after tumor removal.

V: vertebral artery; C: left common carotid artery; E: esophagus; L: longus colli muscle; Th: thyroid

safely dissect the tumor free from the left subclavian and left vertebral arteries and the tumor was removed without opening the parietal or mediastinal pleura (Fig. 2C). Pathologically, resected tumor was diagnosed Schwannoma.

\section{Discussion}

Many approaches for the resection of superior mediastinal tumors have been reported. The approaches have included (1) supraclavicular approach, ${ }^{1)}(2)$ cervico-transsternal approach, ${ }^{2)}(3)$ thoracoscopic surgery (VATS), ${ }^{3)}$ and (4) VATS combined with the supraclavicular approach. ${ }^{4}$ The approach taken is dictated by tumor location with each one having advantages and disadvantages. For instance, tumors bordering the trunk of the brachial plexus or nerve root are best suited for the supraclavicular approach. However, with this approach it is not possible to remove tumors whose distal edge is located beyond Th4. Tumors that extend into the apex are anatomically 
complex and best suited for VATS combined with a supraclavicular approach or a cervico-transsternal approach. VATS itself is minimally invasive and has a superior cosmetic result. ${ }^{4)}$

In orthopedics, a manubrium splitting approach has been employed for fixation of vertebra of the cervicothoracic junction. ${ }^{5}$ We modified this approach and developed an approach which we have called the cervical anterior approach. This approach is only cervical and does not require a sternotomy. Merits of this approach include the ability to remove the tumor without opening the mediastinal or parietal pleura, as well as it obviates the need to drain the thoracic cavity. The tumor is also directly visible, and the surgeon can avoid injury to the great vessels. However, if the tumor extends beyond Th4, it is impossible to see the entirety of the tumors and remove it safety.

We recommend a cervical anterior approach when the tumor is located superior to the Th3 level, when it borders the great vessels, and when it dose not border the trunk of the brachial plexus or nerve root. This approach is easy and safe for surgical procedures.

\section{References}

1) Kohiyama R, Hishikawa S, Meguro H, et al. A case of supramediastinal neurinoma: Its operative approach (via supraclavicular route) and radiographic findings (target sign). Kyobu Geka 1997; 50: 373-6. (in Japanese)

2) Vanakesa T, Goldstraw P. Antero-superior approaches in the practice of thoracic surgery. Eur J Cardiothorac Surg 1999; 15: 774-80 .

3) Arapis C, Gossot D, Debrosse D, et al. Thoracoscopic removal of neurogenic mediastinal tumors: technical aspects. Surg Endosc 2004; 18: 1380-3. Epub 2004 Jun 23 .

4) Akashi A, Ohashi S, Yoden Y, et al. Thoracoscopic surgery combined with a supraclavicular approach for removing superior mediastinal tumor. Surg Endosc 1997; 11: 74-6.

5) Fujimura Y, Nishi Y, Nakamura M, et al. Anterior decompression and fusion for ossification of the posterior longitudinal ligament of the upper thoracic spine causing myelopathy: using the manubrium splitting approach. Spinal Cord 1996; 34: 387-93 . 